\title{
Use of emissive probes in high pressure plasma
}

\author{
Shiluo Yan, Husain Kamal, Jay Amundson, and Noah Hershkowitz \\ Department of Nuclear Engineering and Engineering Physics, University of Wisconsin, Madison, Wisconsin \\ 53706-1687
}

(Received 8 February 1996; accepted for publication 13 August 1996)

\begin{abstract}
The characteristics of emissive probes in unmagnetized high pressure ( $\leqslant 1$ Torr) argon and helium plasmas, produced by inductively coupled $13.56 \mathrm{MHz}$ rf power, are studied. A procedure is given for interpreting emissive probe current-voltage $(I-V)$ characteristics. The $I-V$ curves indicate the amplitude of the rf fluctuation of the plasma potential. They also show ionization near the emissive probe when the potential drop between the emissive probe and the plasma potential is more than the ionization potential. Experiments show that when the temperature of the emissive probe wire and/or the neutral pressure is increased, ionization becomes significant. An increase in the local ion density due to the additional ionization was demonstrated by the $I-V$ curves of an emissive probe and a nearby Langmuir probe. A simple procedure is presented for interpreting these results. (C) 1996 American Institute of Physics. [S0034-6748(96)01111-2]
\end{abstract}

\section{INTRODUCTION}

The plasma potential is an important parameter in most plasma experiments. The $I-V$ characteristic curves of Langmuir probes depend on both the plasma potential and the electron velocity distribution function. When electron tails, plasma drifts, or $\mathrm{rf}$ is present in the plasma, the floating potential of a Langmuir probe is no longer indicative of the plasma potential. ${ }^{1}$ On the other hand, emissive probes have been demonstrated to provide a reliable measurement of the plasma potential at lower pressure ( $\leqslant 10 \mathrm{mTorr}$ ), in the presence of electron tails, plasma drifts, and $\mathrm{rf}^{2-8}$ It has been shown that the floating potential of a strongly heated emissive probe provides an approximation of the plasma potential. ${ }^{9}$ However, as the gas pressure is increased in a weakly ionized plasma, there is a strong tendency for a current discharge to be established near the probe. Electron emission from a strongly heated emissive probe can heavily perturb the local potential near the probe, and in addition, can melt the emissive probe.

The inflection point method of interpreting an emissive probe $^{10}$ is an advance over the floating potential technique because it does not require a strong emission. The inflection point of the $I-V$ curve in the limit of zero emission gives the plasma potential. The inflection point is determined with high accuracy by differentiation of the emissive probe $I-V$ curve. The inflection point is followed as the emission is decreased to zero emission. However, in practice, because the peak of the derivative $d I / d V_{b}$, where $V_{b}$ is the bias voltage, is often small compared to noise and because of the presence of operational amplifier turn-on transients, the inflection point is normally distinguished by its variation with changing emission current. ${ }^{10}$ It has been shown that the potential difference between two peaks in the differentiated $I-V$ trace of an emissive probe gives a good indication of the fluctuation in the plasma potential. ${ }^{11}$ Recently, it has been reported ${ }^{12}$ that a potential structure at the crest and trough phases in an rf oscillation near a powered electrode (for pressure $\leqslant 0.2$ Torr) can be observed and the rf electrode sheath thickness can be estimated using the emissive probe technique given in Ref. 11.
The emissive probe inflection point method in the limit of zero emission has another important advantage. It is the only probe technique that allows measurement of the plasma potential within cathode sheaths where the plasma is nonneutral. To date, measurements have only been carried out at low pressure using this method ${ }^{12,13}$ because of uncertainties in the use of the technique at higher pressure.

As the neutral pressure is increased, it becomes more difficult to determine the plasma potential. At pressures greater than 0.2 Torr, ionization can substantially increase the local plasma density in and around the sheath region because the ionization mean free path becomes comparable to or smaller than sheath dimensions. The perturbed density increases the local plasma potential. ${ }^{14}$ This effect gets worse with increasing electron emission or when the probe is biased far above or far below the plasma potential. The ion and electron currents associated with this increase can far exceed those of the unperturbed plasma. This can be a serious problem when the peak-to-peak rf voltage in the plasma exceeds the ionization potential of the neutral gas so that increased ionization current competes with probe emission current. Application of the inflection point method can be difficult since the inflection point is normally distinguished by its variation with changing emission current. Nevertheless, in this article, we show how to use the inflection point technique to detect the plasma potential in the presence of rf at pressures as high as 1 Torr.

\section{EXPERIMENTAL APPARATUS}

The experimental apparatus is shown in Fig. 1. The relative position, orientation, and size of the electric probes are also presented. The system consists of a cylindrical vacuum chamber with a roughing pump. The neutral gas pressure in the chamber was varied from 10 mTorr to 1 Torr. A one turn ring antenna in the vacuum chamber is insulated from the plasma with fiber glass. The $13.56 \mathrm{MHz}$ rf power supply is connected to the antenna using a capacitive matching circuit which is adjusted to minimize the reflected power.

A movable Langmuir probe with a 1/4 in. diam onesided disk was used to measure the $I-V$ characteristic curve. 


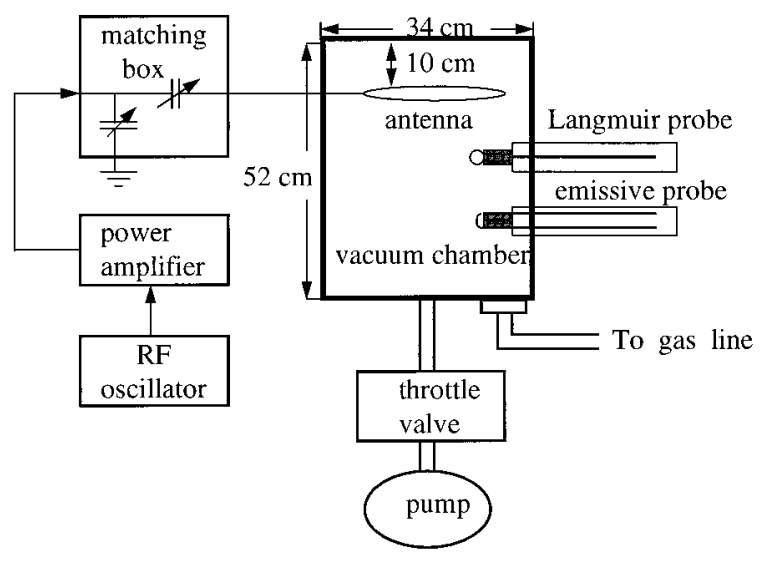

FIG. 1. The experimental set up.

The $I-V$ curve was then used to calculate the basic parameters of the plasma. The filaments of the emissive probe are of thoriated tungsten, $0.0025 \mathrm{~cm}$ in diameter and 0.5 to 1.0 $\mathrm{cm}$ long. The filaments are spot welded to gold coated copper wires and insulated with ceramic. The probe shafts are constructed of $8 \mathrm{~mm}$ stainless steel tubing with coaxial ceramic tubing for insulation. BNC connectors are used for the emissive filament heating and bias voltage connections.

A schematic diagram of the circuits used for this study is shown in Fig. 2. A dc heating current was supplied by a floating power supply which consisted of an isolation amplifier with a buffer output as shown in Fig. 3. The heating voltage could easily be controlled by increments of $0.01 \mathrm{~V}$ at the $D / A$ output channel. Both the heating voltage and the bias voltage of the emissive probe were computer controlled. Another $D / A$ output channel was used to produce a sawtooth voltage with a range of -5 to $+5 \mathrm{~V}$, which was amplified by an operational amplifier to be -100 to $+100 \mathrm{~V}$. A resistor voltage divider was used to transfer the sweep voltage to an $A / D$ input channel to monitor the actual sweep voltage. A current sampling resistor with a capacitive filter was used to convert the probe current to voltage and to transfer the col-

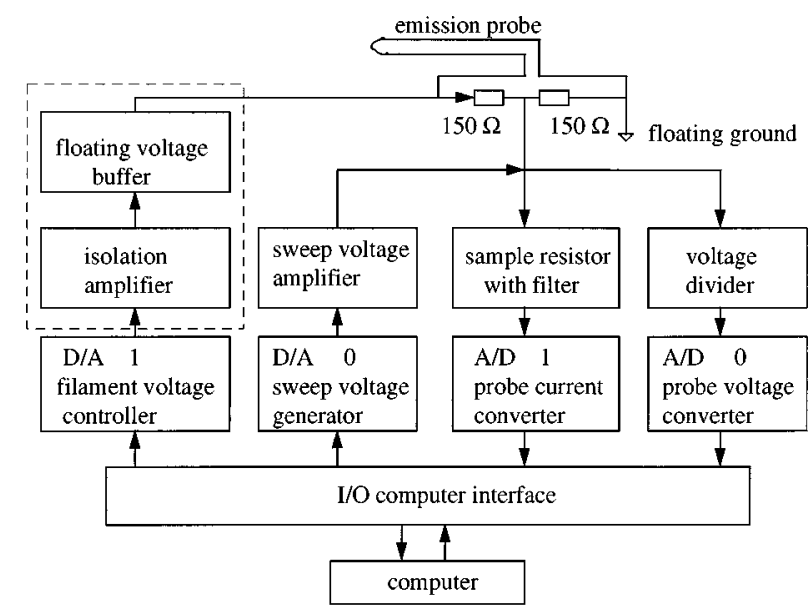

FIG. 2. A schematic diagram of the emissive probe circuits.

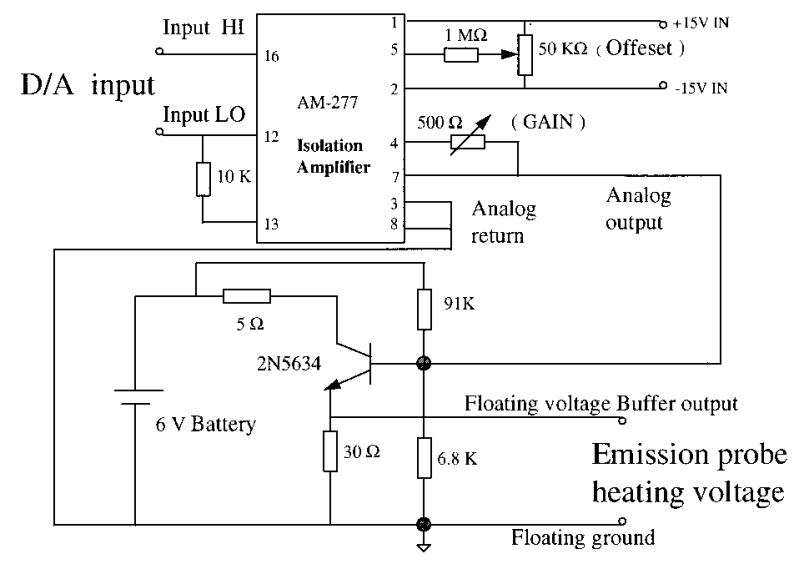

FIG. 3. A detailed schematic of the isolation amplifier with the buffer output shown in Fig. 2.

lected voltage to another $A / D$ input channel. This set up allowed real time data collection.

The data were extracted and corrected by a data processing program. The procedure was (1) collecting the $I-V$ data with rf plasma, (2) collecting the $I-V$ data without $\mathrm{rf}$ power (i.e., the load line), (3) smoothing both sets of data, (4) determining the slope and the shift of the load line, and (5) reproducing the "pure" $I-V$ curve by subtracting the load line data. These data were reproducible and gave a reliable measurement of the probe $I-V$ characteristics.

The same circuits and procedure was used to obtain the $I-V$ curve of a collecting Langmuir probe (without supplying a heating voltage). From a semilog $I-V$ characteristic plot, the electron temperature was determined from the slope of the $I-V$ curve near the knee at the minimum value of the fluctuating plasma potential. This required identification of the minimum value of the plasma potential. Once the temperature and ion saturation current were known, the plasma density was calculated using the Bohm current formula. ${ }^{15}$

\section{EXPERIMENTAL RESULTS AND DISCUSSION}

A representative $I-V$ trace of an emissive probe in argon plasma at 0.5 Torr neutral pressure for small rf power $(10 \mathrm{~W})$ is shown in Fig. 4. The data exhibit a number of features. The points $D$ and $E$ are obtained from the differentiated $I-V$ trace corresponding to Fig. 4, given in Fig. 5. The segment $D E$ is of major importance. It represents the plasma potential fluctuation amplitude. The midpoint of $D$ and $E$ gives a time averaged plasma potential. The linear segments $F G$ and $B C$ have been identified as the electron saturation current and the temperature limited emissive current, respectively. These emissive probe characteristics generally can be found at low pressure. The nonlinear segments $G H$ and $A B$ are a more interesting phenomena in high pressure discharges. Both lines deviate from the continuation of lines $F G$ and $B C$ (which are shown as dashed lines) can be explained as ionization currents. The points $B$ and $G$ are from the onset of ionization. $G H$ is produced when the emissive probe is biased to a sufficiently large positive bias and $A B$ occurs when it is biased to a sufficiently negative bias with respect to the plasma potential. The midpoint of $B$ and $G$ 


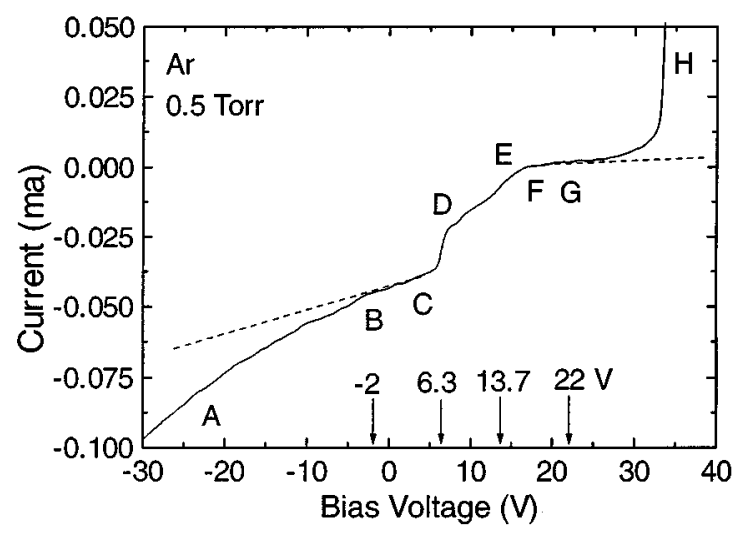

FIG. 4. The $I-V$ curve of the emissive probe for Ar plasma at 0.5 Torr. The interesting regions are labeled $A-H$. The short arrows point to the onsets of ionization and the fluctuating plasma potential.

also gives a time averaged plasma potential which is in agreement with the value determined by the inflection point method.

The details of the differentiated curve in the region between $C$ and $F$ are shown in Fig. 6. These data resemble data shown in Ref. 3 and allow identification of the difference voltage between the two peaks as a measure of the plasma potential fluctuation amplitude. ${ }^{11}$ This hypothesis was checked by varying the rf power. As seen in Fig. 7, the difference between the two peaks increases approximately linearly with increasing rf power (with a zero power intercept approximately equal to zero).

The variation in the inflection point potential with emission current is given in Fig. 8 for rf power equal to $15 \mathrm{~W}$ and neutral pressure equal to 0.5 Torr. These data show that as emission is decreased, the upper $\left(V_{\mathrm{PH}}\right)$ inflection point moves to higher potentials while the lower $\left(V_{\mathrm{PL}}\right)$ inflection point moves to lower potentials. The time average plasma potential, defined as the potential midway between the two inflection points, is approximately constant with emission current. Also note, the probe behavior shown in Fig. 8 is similar to those previously found at much lower pressure. ${ }^{11}$

For the data shown in Fig. 6, the minimum plasma potential $\left(V_{\mathrm{PL}}\right)$ of peak $D$ is $6.3 \mathrm{~V}$, and the maximum plasma

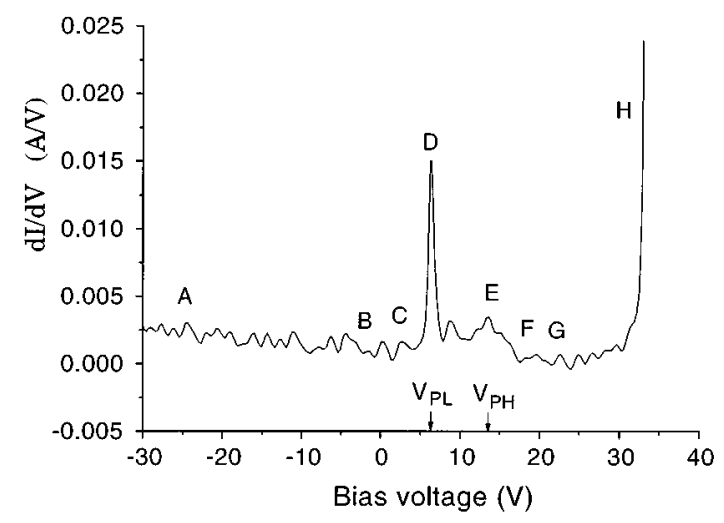

FIG. 5. The derivative of the current vs bias voltage of the data given in Fig. 4. The two peaks of the plasma potential are labeled.

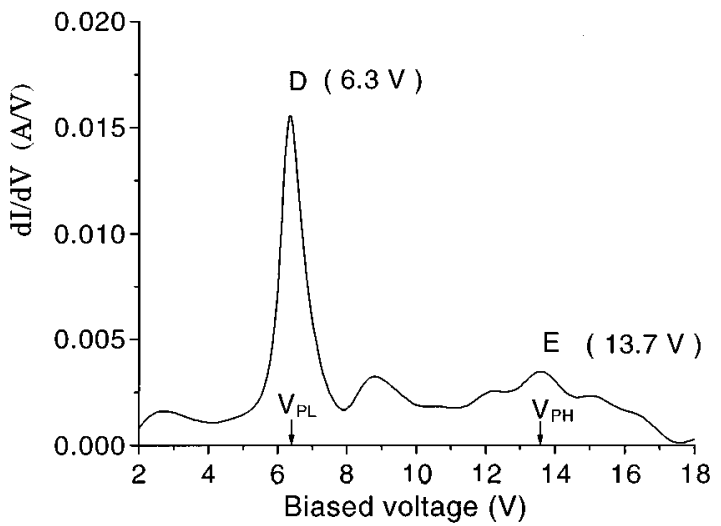

FIG. 6. A selected range of the $I-V$ curve in Fig. 5. Notice the fluctuating plasma potential $V_{\mathrm{PL}}$ and $V_{\mathrm{PH}}$.

potential $\left(V_{\mathrm{PH}}\right)$ of peak $E$ is $13.7 \mathrm{~V}$. Therefore, the amplitude of the fluctuation is approximately $7 \mathrm{~V}$. The midpoint of these two peaks $(10 \mathrm{~V})$ is the time averaged plasma potential $V_{p} .{ }^{11}$ Our experimental data also indicate that the time averaged plasma potential increases with increasing sinusoidal rf power. In Fig. 9, the two curves represent the results for argon plasmas at neutral pressures of 0.1 and 0.75 Torr, respectively. The data in Fig. 9 show that the plasma potential becomes more positive as the neutral pressure increases for the same rf power.

Figure 4 also shows that segments $F G$ and $G H$, with bias voltages $V_{b}>\left(V_{\mathrm{PH}}\right)$, contain contributions from the electron collection current. The plateau segment $F G$ corresponds to the usual probe electron saturation current. However, the electron current is observed to increase as the positive bias voltage is increased. This is plotted as segment $G H$. This increase in electron current can be explained by additional ionization when the bias voltage is more positive than the minimum value of the fluctuating plasma potential. The onset of ionization occurs at a bias voltage of approximately $22 \mathrm{~V}$ at $G$, while the minimum fluctuating potential $\left(V_{\mathrm{PL}}\right)$ is approximately $6.3 \mathrm{~V}$. The difference in potential is then approximately equal to the ionization potential of the argon gas $(15.8 \mathrm{~V})$, so plasma electrons can acquire enough energy to

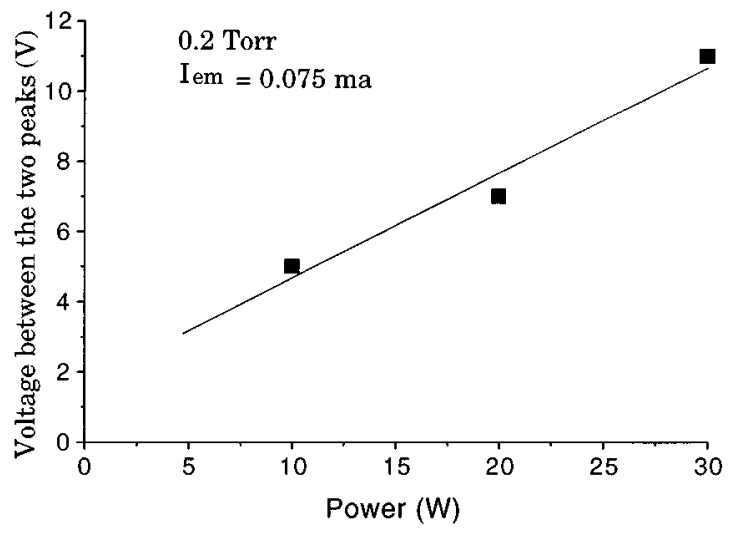

FIG. 7. The voltage between $V_{\mathrm{PH}}$ and $V_{\mathrm{PL}}$ of the emissive probe as a function of rf power. 


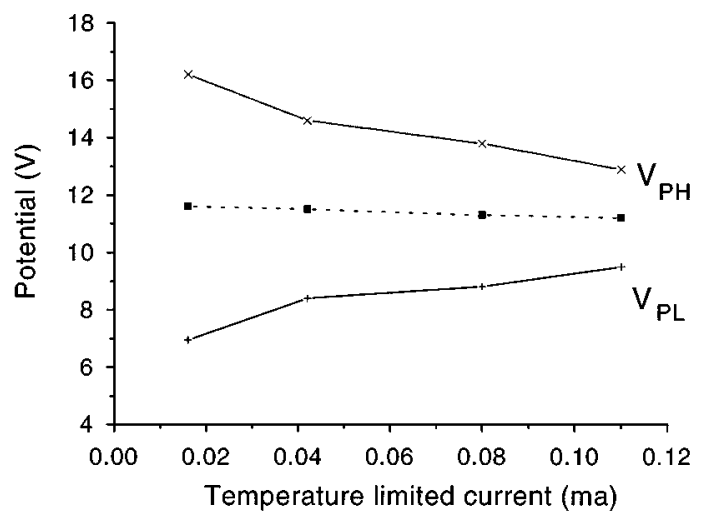

FIG. 8. $V_{\mathrm{LP}}, V_{\mathrm{LH}}$, and the time average plasma potential as a function of the increase in the temperature limited current.

ionize the gas in going from the background plasma to the probe.

Electron emission is not critical to the sudden increase in electron current seen with emissive probes as shown in Fig. 4. In fact, the same phenomenon was observed in the $I-V$ trace of a Langmuir probe as shown in Fig. 10. The experimental conditions are approximately the same as in Fig. 4. Note that again the current begins to increase at approximately $22 \mathrm{~V}$.

When the $\log (I)$ is graphed vs bias voltage $V_{b}$ (see Fig. 11), knees are apparent at about $6.3 \mathrm{~V}$ and another at about $26 \mathrm{~V}$. The lower knee corresponds to the minimum plasma potential $V_{\mathrm{PL}}$ (labeled $D$ in Figs. 4-6) identified by the emissive probe. The knee at around $26 \mathrm{~V}$ can then be identified as a consequence of ionization as it was for the emissive probe.

By fitting a line to the slope of $\log (I)$ vs $V_{b}$ on the semilog plot Fig. 11, the electron temperature can be calculated using: ${ }^{13}$

$$
T_{e}=e \frac{d V_{b}}{d \ln (I)} .
$$

The plasma electron density was determined from the ion saturation current (see Fig. 12) using:

$$
I_{\mathrm{sat}}=\frac{1}{2} n_{e} A \sqrt{\frac{T_{e}}{m_{i}}} .
$$

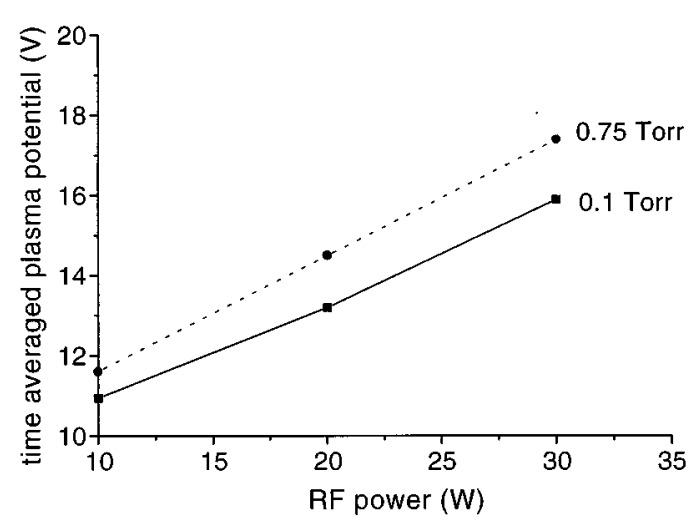

FIG. 9. Time averaged plasma potential as a function of the rf power.

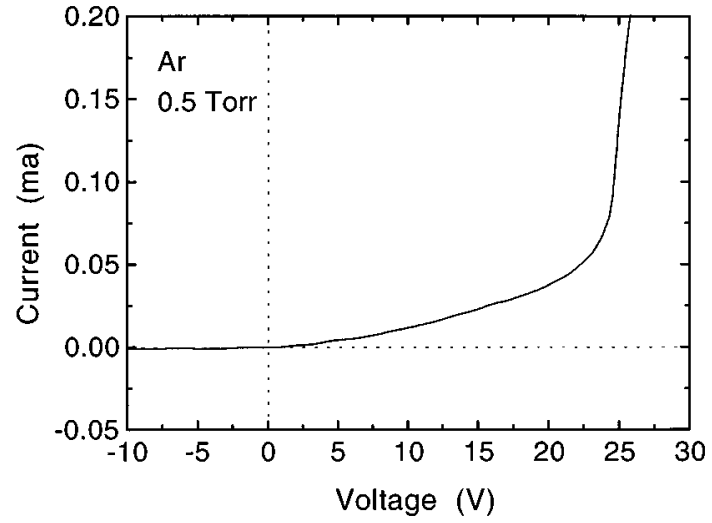

FIG. 10. $I-V$ curve of the Langmuir probe.

Here $I_{\text {sat }}$ is the ion saturation current, $n_{e}$ is the electron plasma density, $T_{e}$ is the electron temperature, $A$ is the probe disc area, and $m_{i}$ is the ion mass. The electron temperature and the electron density are $1.4 \mathrm{eV}$ and $2.1 \times 10^{8} \mathrm{~cm}^{-3}$, respectively, for Figs. 4 and 11.

In this way, we also measured the electron temperature and the electron density as a function of neutral gas pressure, at the center of the chamber (see Figs. 13 and 14). Figure 13 shows that the electron temperature decreases with increasing gas pressure. Figure 14 shows that the electron density increases with increasing gas pressure.

In Fig. 4, a short straight line $B C$ for a temperature limited emissive current is observed. This is due to the finite wire temperature. However, it was observed that the emissive probe current was not limited when the emissive probe was biased more negatively. When the emissive probe was biased negative, with respect to the maximum plasma potential $\left(V_{\mathrm{PH}}=13.7 \mathrm{~V}\right)$, by an amount greater than the ionization potential of argon $\left(\epsilon_{i}=15.8 \mathrm{~V}\right)$, additional ion current from ionization was produced shown as segment $A B$ in Fig. 4. Also in Fig. 4, the onset of ionization was at approximately $-2 \mathrm{~V}$ at $B$. Therefore, the presence of the onset of ionization for both positive and negative bias voltages provides another way to determine the time average plasma potential with an emissive probe. In Fig. 4 the midpoint of $B$ and $G$ gives the time averaged plasma potential $V_{p}=10 \mathrm{~V}$, in agreement with

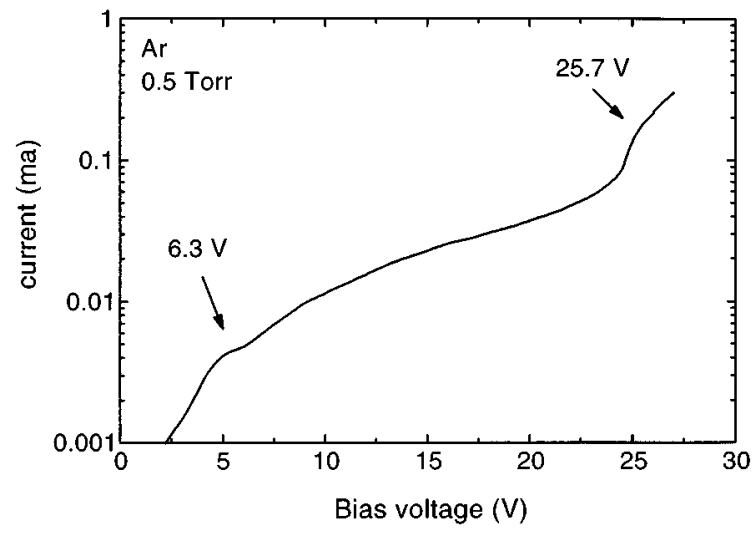

FIG. 11. Semilog plot of the Langmuir probe $I-V$ curve in Fig. 10. 


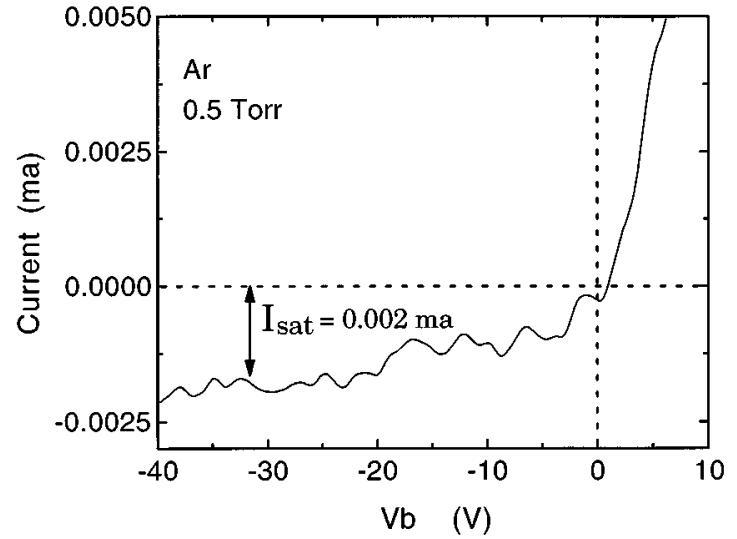

FIG. 12. A detail of the Langmuir probe $I-V$ curve in Fig. 10.

the value determined by the inflection point method, with $D$ and $E$ in Fig. 5.

When the emissive probe is biased negatively with respect to the $V_{\mathrm{PH}}$, the electrons emitted from the filament are accelerated to the plasma potential. This gives the electrons sufficient energy to ionize the background gas if the potential difference exceeds the ionization potential. The ionization is restricted to a region within the order of the ionization mean free path $\lambda_{i}$. At high neutral pressure, the size of this region can become comparable to the sheath dimension. The ionization increases the plasma density near the emissive probe with a corresponding increase in the ion saturation current to the probe. The ion saturation current also increases with the emissive probe wire temperature. Figures 15 and 16 show $I-V$ characteristics of an emissive probe at four probe temperatures for helium and argon plasmas, respectively. When the temperature of the wires increases, the ionization becomes significant at smaller negative bias voltages. The data also show that the onset of ionization occurs at a lower bias voltage in argon than in helium, consistent with the ionization potential $\epsilon_{i}$ of argon and helium gas (15.8 and $24.6 \mathrm{eV}$, respectively). ${ }^{16}$

Figure 17 shows the $I-V$ curves of an emissive probe at approximately the same wire temperature but at different pressures. These curves correspond to approximately the

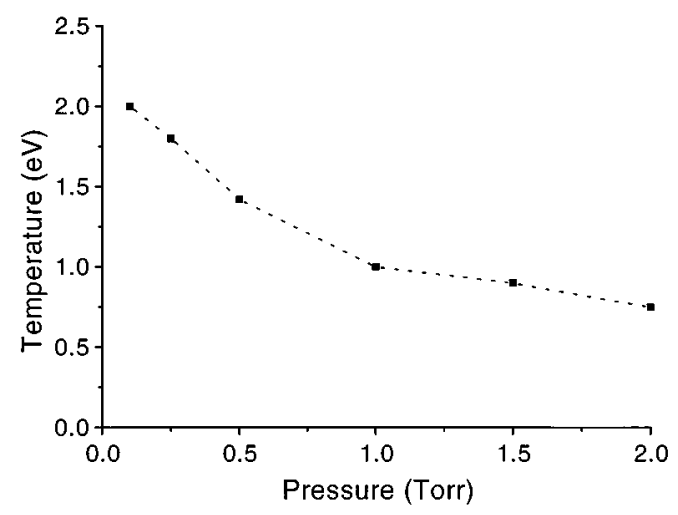

FIG. 13. Electron temperature of Ar plasma as a function of pressure in the middle of the chamber.

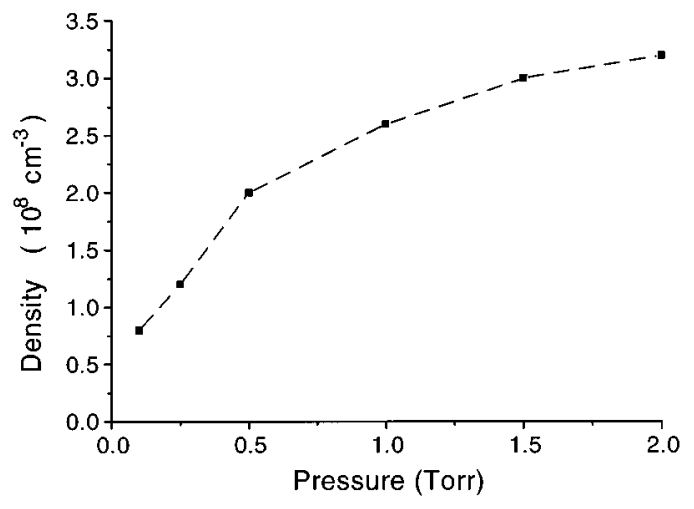

FIG. 14. Electron density of Ar plasma as a function of pressure in the middle of the chamber.

same temperature limited emissive current $\left(I_{e m}\right)$ with different ionization currents $\left(\Delta I_{i}\right)$. The ionization currents all increase with neutral pressure when the emissive probe is more positively or negatively biased than the ionization potentials with respect to the plasma potential. It is apparent that the $I-V$ curves of the emissive probe are not symmetric at positive and negative bias voltages.

The asymmetry is present because the ionization currents have different origins. Although both increases are the result of increased ionization, the increased negative current in the negative bias ionization region is due to extra ion current, while the increased positive current in the positive bias ionization region is due to increased electron collection. In addition, the ionization takes place in different locations. For the negative bias, electrons emitted at the probe are the source of the additional ionization. For the positive bias, the additional ionization takes place within the sheath where plasma electrons gain energy as they accelerate towards the probe wire.

In the negative bias case, the enhanced ionization rate due to the emitted electrons is in the order of $I_{e m} / e$. Since sufficient energy is not available for multiple ionization per electron and the ionization takes place within a region the order of one mean free path $\lambda_{i}$ of the emissive probe wire.

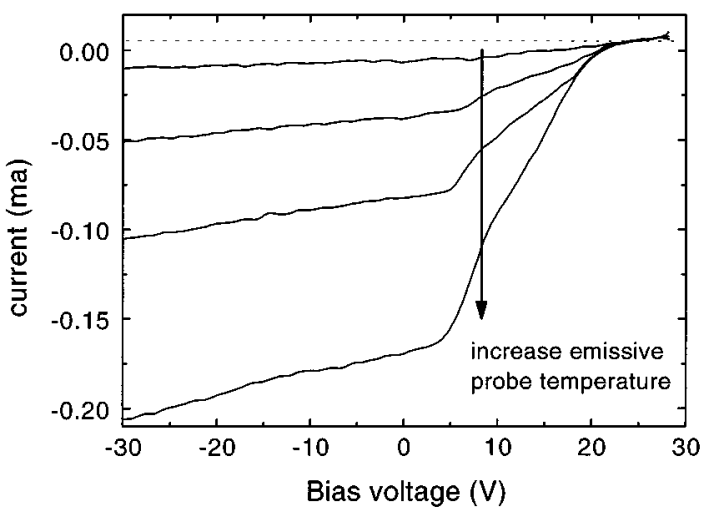

FIG. 15. $I-V$ curve of the emissive probe for He plasma at four probe wire temperatures at 0.5 Torr. 


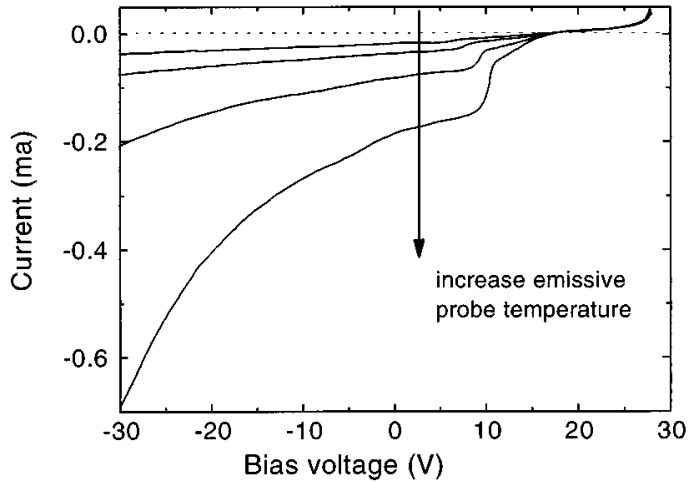

FIG. 16. $I-V$ curves of the emissive probe for Ar plasma at four probe wire temperatures at 0.5 Torr.

Ions are lost at the sheath boundary and at the boundary of the enhanced ionization region, with enhanced loss rates given approximately by $\Delta n c_{s} \pi s L$ and $\Delta n c_{s} \pi \lambda_{i} L$, respectively, see Fig. 18. Here $s$ is the sheath thickness, $L$ is the wire length, $\Delta n$ is the enhanced ion density, and $c_{s}$ is the ion sound velocity.

The total particle flux is still conserved. In equilibrium, the total ion loss current equals $I_{\mathrm{em}}$. The total current $I$ is given by

$$
I=I_{\mathrm{em}}+\Delta I_{i}
$$

where, $I$ is the measured current by the emissive probe, $I_{e m}$ is the emitted electron current, and $\Delta I_{i}$ is the portion of ion current (due to the ionization by $I_{\mathrm{em}}$ ) collected by the probe rather than lost to the outer boundary of the ionization region, as in Fig. 18. Assuming that most of the ionization takes place outside the sheath (assuming $s \ll \lambda_{i}$ ) the increased ion current $\Delta I_{i}$ was given by

$$
\Delta I_{i}=2 \pi s L I_{\mathrm{em}} / 2 \pi\left(s+\lambda_{i}\right) L \approx I_{\mathrm{em}} s / \lambda_{i}=I_{\mathrm{em}} s n_{0} \sigma(\epsilon),
$$

where,

$$
\begin{array}{ll}
\epsilon=e\left(V_{\mathrm{PH}}-V_{b}\right), & V_{b}<0, \\
\epsilon=e\left(V_{b}-V_{\mathrm{PL}}\right), & V_{b}>0,
\end{array}
$$

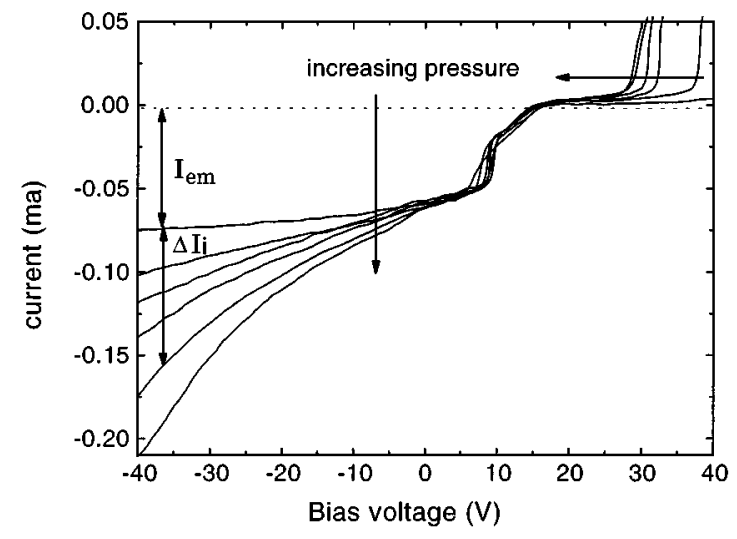

FIG. 17. $I-V$ curves of the emissive probe with the same wire temperature but at different pressures (0.05-1 Torr).

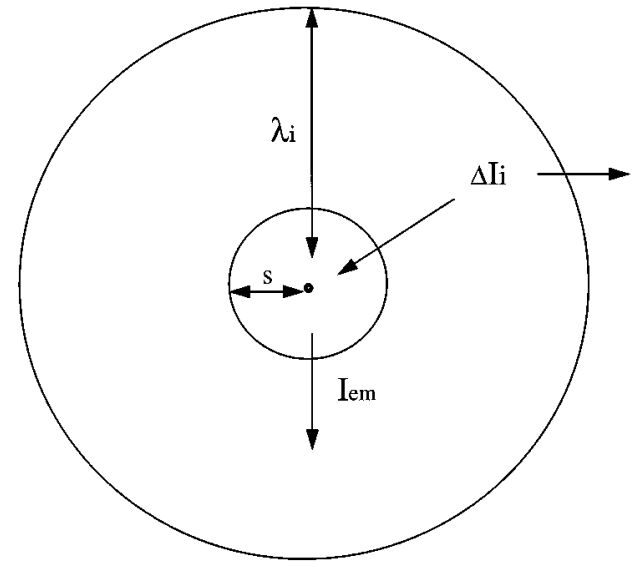

FIG. 18. A schematic of the region near the emissive probe wire, at high pressure. $I_{\mathrm{em}}$ is the emitted electron current from the wire and $\Delta I_{i}$ is the fraction of ion current falling into the sheath.

where $n_{0}$ is the neutral density and $\sigma(\epsilon)$ is the ionization cross section at electron energy $\epsilon$. From Eq. (4), we note that the enhanced ion current $\Delta I_{i}$ is proportional to $1 / \lambda_{i}=\sigma(\epsilon) n_{0}$. If the pressure is increased, $\lambda_{i}$ becomes smaller and $\Delta I_{i}$ increases as in Fig. 17. This is because ionization occurs closer to the sheath, therefore a larger fraction of the ions fall through the sheath. The results given in Fig. 19 show that after dividing each of the curves in Fig. 17 by the appropriate $s / \lambda_{i}$, all curves coalesce to a single curve corresponding to the lowest pressure in Fig. 17. This demonstrates the additional current is proportional to $\sigma(\epsilon) n_{0}$ assuming the bulk electron temperature keeps constant during emission.

On the other hand, when the probe is strongly biased positive, the plasma electrons inside the sheath have higher electron energy than that outside the sheath. The additional ionization occurs inside the sheath region. This process produces significant ionization if the $\lambda_{i}$ is comparable to the sheath thickness. The electrons from the enhanced ionization are directly collected by the positively biased probe. Thus, ionization effects are more apparent at positive bias.

The increase of the plasma density near the emissive probe, due to the ionization, is measured by a nearby Lang-

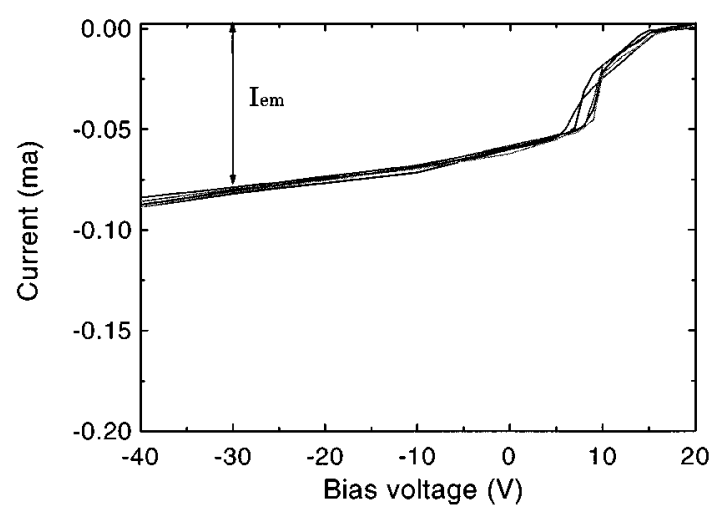

FIG. 19. The emissive probe $I-V$ curves after dividing each of the curves in Fig. 17 by the appropriate $s / \lambda_{i}$. 


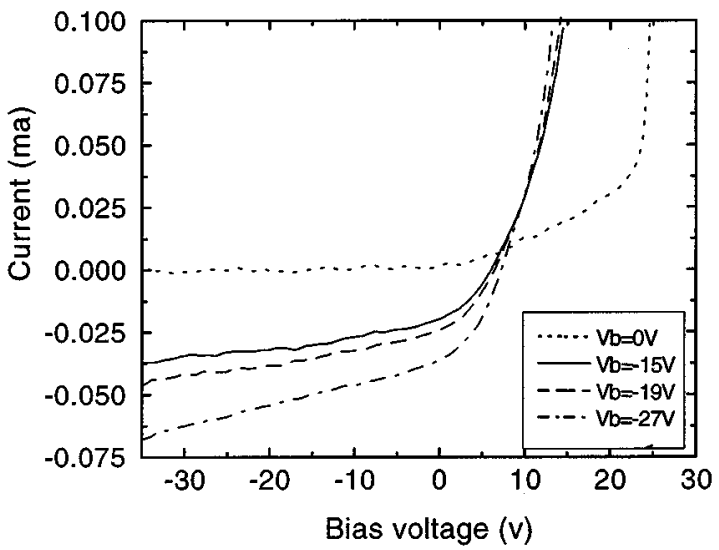

FIG. 20. The $I-V$ curve of the Langmuir probe near the emissive probe $(\approx 1$ $\mathrm{cm}$ ) for Ar plasma at 0.5 Torr. Notice the increase in $I_{\text {sat }}$ due to the increase in ionization as $V_{b}$ becomes more negative.

muir probe. The changes in the $I-V$ curve of the nearby Langmuir probes for argon and helium plasma with different negative bias voltages, applied to the emissive probe, are shown in Figs. 20 and 21, respectively. The data show a larger required potential difference for $\mathrm{He}$ compared to $\mathrm{Ar}$ because of the difference in the ionization potential.

Figure 22 compares the increase in the ion current $\Delta I_{i}$ for argon plasma, measured by the emissive probe, to the ion saturation $I_{\text {sat }}$ current measured by the nearby Langmuir probe. Figure 23 is the same as Fig. 22 but for helium gas. Thus, it is apparent that the increase in ion current at the emissive probe is a result of an increase in the local ion density.

Because of the presence of ionization, emissive probes at high pressure provide several techniques to determine the plasma potential. These include fluctuating plasma potential from inflection point method and also from the onset of ionization.

\section{PROCEDURE FOR INTERPRETING HIGH PRESSURE DISCHARGES DATA}

When measuring the plasma potential using the inflection point method, one should make sure to bias the probe

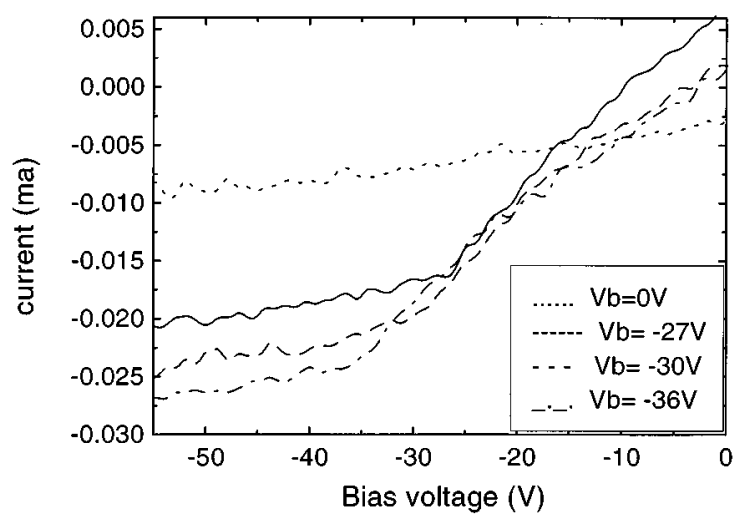

FIG. 21. The $I-V$ curve of the Langmuir probe near the emissive probe $(\approx 1$ $\mathrm{cm}$ ) for He plasma at 0.5 Torr. As the $V_{b}$ of the emissive probe becomes more negative, $I_{\mathrm{em}}$ increases due to increased ionization.

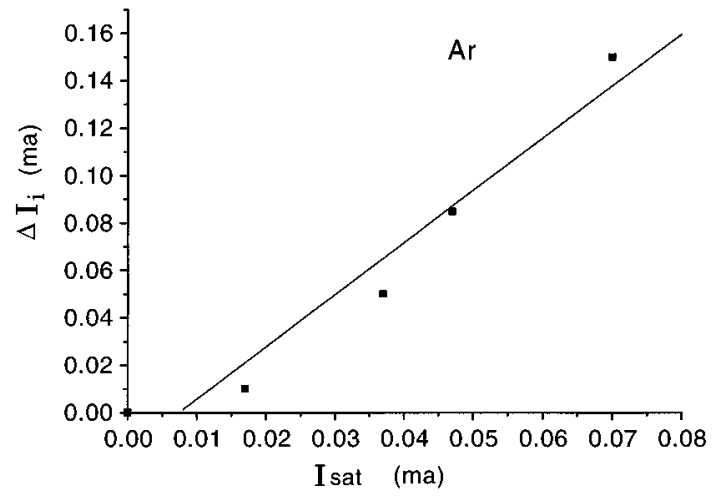

FIG. 22. The ion saturation current measured by the Langmuir probe, near the emissive probe $(\approx 1 \mathrm{~cm})$, as a function of the increase in the ion current measured by the emissive probe for Ar plasma at 0.5 Torr.

close to the region where the inflection occurs. This is to minimize the heating time and to avoid the excess current due to the excess ionization near the wire. The measured currents in this method should be less than $1 \mathrm{~mA}$.

The following steps are suggested to measure the plasma potential in a plasma with $0.1<p<1$ Torr, using an emissive probe:

(1) Slowly increase the dc heating voltage until the emissive wire starts to glow white.

(2) Sweep the probe bias from -100 to $+100 \mathrm{~V}$ in order to make sure that all of the ionization features are included.

(3) Plot the $I-V$ curve and look for the two bias voltages where the current begins to deviate from the ion and electron saturation current (see points $B$ and $G$ in Fig. 4). Subtract the ionization energy $\left(\epsilon_{i}\right)$ of the gas from the upper voltage and add $\epsilon_{i}$ to the lower voltage to get approximate value for $V_{\mathrm{PL}}$ and $V_{\mathrm{PH}}$.

(4) Sweep the probe with a shorter range bias voltage, for example from $V_{\mathrm{PL}}-20 \mathrm{~V}$ to $V_{\mathrm{PH}}+20 \mathrm{~V}$. This reduces the wire heating time and protects the circuits from measuring unnecessarily large current due to ionization near the probe.

(5) To get a more accurate measurement of the plasma po-

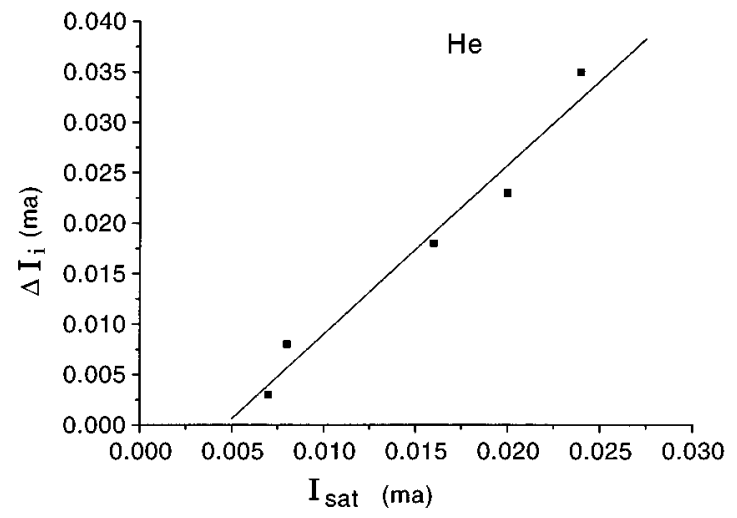

FIG. 23. The ion saturation current measured by the Langmuir probe, near the emissive probe $(\approx 1 \mathrm{~cm})$, as a function of the increase in the ion current measured by the emissive probe for He plasma at 0.5 Torr. 
tential use the inflection point method. If the inflection point in the $I-V$ curve is not apparent at $V_{\mathrm{PL}}$ and $V_{\mathrm{PH}}$, increase the dc heating voltage of the emissive wire by small increments until the inflection point appears in the $I-V$ curve.

(6) Take the derivative of the current with respect to the bias voltage to look for the two peaks of the fluctuating plasma potentials $V_{\mathrm{PL}}$ and $V_{\mathrm{PH}}$.

(7) Decrease the emissive wire temperature by steps of 0.01 $\mathrm{V}$ until the two peaks in step (6) are not detectable. This step is to insure finding the plasma potential at the limits of zero emission. ${ }^{10}$

${ }^{1}$ S. Lizuka, P. Michelsen, J. J. Rasmussen, R. Schrittwieser, R. Hatakeyama, R. K. Eaeki, and N. Sato, J. Phys. E 14, 1291 (1981).

${ }^{2}$ N. Hershkowitz, B. Nelson, J. Pew, and D. Gates, Rev. Sci. Instrum. 54, 29 (1983).

${ }^{3}$ F. F. Chen, in Plasma Diagnostic Techniques, edited by R. H. Huddle- stone and S. L. Leonard (Academic, New York, 1956), p. 113.

${ }^{4}$ E. Y. Wang, T. Intrator, and N. Hershkowitz, Rev. Sci. Instrum. 56, 519 (1985).

${ }^{5}$ H. Yamada, and D. Murphree, Phys. Fluids 14, 1120 (1970).

${ }^{6}$ C. Forest and N. Hershkowitz, J. Appl. Phys. 60, 1295 (1986)

${ }^{7}$ M. H. Cho, N. Hershkowitz, and T. Intrator, J. Vac. Sci. Technol. A 6, 2978 (1988).

${ }^{8}$ Y. Okuno, Y. Ohtso, and H. Fujita, Phys. Lett. 193, 94 (1994).

${ }^{9}$ R. E. Kemp and J. M. Sellen, Rev. Sci. Instrum. 37, 455 (1966)

${ }^{10}$ J. R. Smith, N. Hershkowitz, and D. Coakley, Rev. Sci. Instrum. 50, 210 (1979).

${ }^{11}$ E. Y. Wang, N. Hershkowitz, T. Intrator, and C. Forest, Rev. Sci. Instrum. 57, 2425 (1986).

${ }^{12}$ Y. Okuno and H. Fujita, Appl. Phys. 70, 642 (1991).

${ }^{13}$ N. Hershkowitz, Plasma Diagnostics, edited by Orlando Auciello and Daniel L. Flamm (Academic, San Diego, 1989), p. 113.

${ }^{14}$ W. B. Pennebaker, IBM J. Res. Dev. 23, 16 (1979).

${ }^{15}$ F. F. Chen, Introduction to Plasma Physics and Controlled Fusion, 2nd ed. (Plenum, New York, 1984).

${ }^{16}$ B. Chapman, Glow Discharge Processes Sputtering and Plasma Etching, (Wiley, New York, 1980). 